\title{
Dispersive contribution to the nucleus-nucleus potential
}

\author{
M. Lozano \\ Departamento de Física Atómica y Nuclear, Facultad de Física, 41080 Sevilla, Spain
}

(Received 24 February 1987)

\begin{abstract}
With a simple parametrization of the empirical imaginary potential depths, the real potential induced by a dispersion relation is calculated analytically for the elastic scattering of ${ }^{16} \mathrm{O}$ by several target nuclei. Between $25-50 \%$ of the real potential empirical depths can be attributed to this dispersive contribution when the energy approaches the Coulomb barrier. Thus, the anomaly observed in the microscopically calculated real potential at these energies can be explained by taking into account the real part induced from the imaginary one through the dispersion relation.
\end{abstract}

The energy dependence of the nucleus-nucleus optical potential is not well established so far. From an empirical point of view the ambiguity of the parameter sets of simple forms, determined from the analysis of experimental data, makes it difficult to obtain clear information about their energy dependence. ${ }^{1}$ One of the best microscopic methods to calculate the real potential is the double folding procedure by using a realistic effective interaction. This can be derived from $G$-matrix elements based on the Reid potential ${ }^{2}$ or it can be built for computing the general properties of spherical nuclei. ${ }^{3}$ In both cases the double folding potential has not any energy-dependent term. The variation with the energy of the imaginary part is better known from some empirical analyses (see, for instance, Refs. 4-6) or from some phenomenological models). ${ }^{7}$ In general, the optical potential constructed with a double folding real potential and an empirical or phenomenological imaginary part allows us to reproduce fairly well the elastic scattering experimental data for a wide range of masses and energies. There are two general exceptions. The first one is the case of the scattering of light-heavy ions, such as ${ }^{6,7} \mathrm{Li}$ and ${ }^{9} \mathrm{Be}$, where the folded potential has to be reduced almost $50 \%$ in order to fit the data. $^{2}$ In these cases a detailed study of the reaction mechanisms is needed to explain the necessity of such strong renormalization of the real potential (see Ref. 8 and references therein). The second failure of the folding potential has been reported more recently, mainly for the system ${ }^{16} \mathrm{O}+{ }^{208} \mathrm{~Pb}$ at energies close to the Coulomb barrier ${ }^{9}$ and for the system ${ }^{32} \mathrm{~S}+{ }^{40} \mathrm{Ca}$ at three different energies. ${ }^{10}$ In principle, the anomalies of the potential can be attributed to couplings of the elastic scattering with nonelastic channels that could be described by explicit coupled-channel calculations. ${ }^{11-13}$ As an alternative, Nagarajan, Mahaux, and Satchler have suggested ${ }^{14}$ including all possible couplings in a dispersion relation. Thus, with an energy-dependent imaginary part an energydependent real term can be obtained through the dispersion relation. The strong contribution of this term at energies close to the barrier and at the relevant distances for the elastic scattering can explain the observed anomalies of the optical potential. Some extensions of this dispersion relation approach to the extrapolation towards negative energy ${ }^{15}$ and to the nucleus-nucleus potential of a variety of systems ${ }^{16}$ have been made recently.

In this work the energy-dependent contribution to the real potential that arises from the imaginary potential through the dispersion relation is estimated. A simple linear parametrization of the empirical imaginary depths is assumed for the quantitative determination. The addition of such a term to the microscopically calculated real potential can avoid the necessity of renormalizing that potential in order to fit the elastic scattering experimental data at energies close to the Coulomb barrier.

The nucleus-nucleus optical potential can be expressed in a local and angular momentum independent form as

$$
U(r, E)=V(r)+\Delta V(r, E)+i W(r, E),
$$

where $\Delta V(r, E)$ is the "dispersive" term arising from the absorptive potential $W(r, E)$ through the dispersion relation

$$
\Delta V(r, E)=\frac{P}{\pi} \int_{0}^{\infty} \frac{W\left(r, E^{\prime}\right)}{E-E^{\prime}} d E^{\prime} .
$$

In principle, the real potential $V(r)$ must coincide with the microscopic potential and the sum $V(r)+\Delta V(r, E)$ must coincide with the empirical potential that reproduces the elastic scattering experimental data.

In a recent work $^{7}$ we proposed from phenomenological considerations an imaginary potential of the form

$$
W(r, E, L)=W(E, L) W(r),
$$

where the geometrical part $W(r)$ is parametrized as

$$
W(r)=\left\{1+\exp \left[\left(r-R_{i}\right) / a_{i}\right]\right\}^{-1},
$$

with $R_{i}=r_{i}\left(A_{1}^{1 / 3}+A_{2}^{1 / 3}\right)$, and the energy- and angularmomentum-dependent depth of the form

$$
\begin{aligned}
W(E, L) & =0 \text { if } E^{*}<E_{y}(L) \\
& =c \rho\left(E^{*}, L\right) \text { if } E_{y}(L)<E^{*}<E_{y}(L)+E_{1} \\
& =c_{0}+c_{1} E \text { if } E^{*}<E_{y}(L)+E_{1},
\end{aligned}
$$

where $\rho\left(E^{*}, L\right)$ is the compound nucleus level density, $E_{y}(L)$ is the yrast energy, $E^{*}$ is the excitation energy, and $E_{1}, c_{0}$, and $c_{1}$ are the constants of the model. A numeri- 


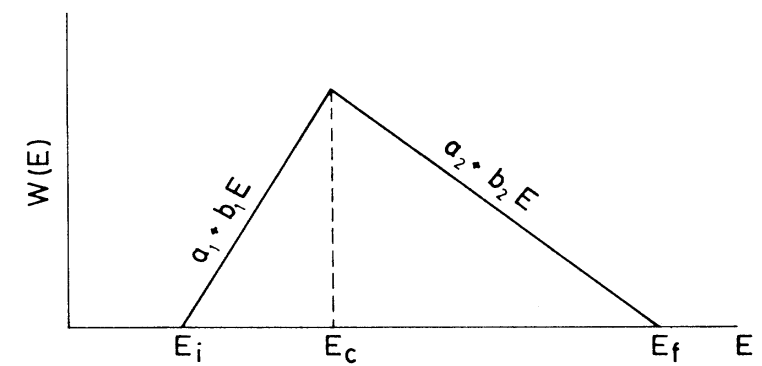

FIG. 1. Scheme of the straight-line fit to the empirical imaginary potential depths.

cal evaluation of the dispersion relation using this potential will be published elsewhere. In order to obtain an analytical expression of the dispersive potential, expression (5) can be simplified along the same line as has been done by Mahaux, Ngô, and Satchler, ${ }^{16}$ in the schematic form

$$
\begin{aligned}
W(E) & =0 \text { if } E<E_{i} \\
& =a_{1}+b_{1} E \text { if } E_{i}<E<E_{c} \\
& =a_{2}+b_{2} E \text { if } E_{c}<E<E_{f}
\end{aligned}
$$

where the meaning of the constants is clear in Fig. 1. The evaluation of the dispersion relation (5) for the imaginary potential $W(r, E)$ constructed with a geometrical factor as expressed by Eq. (4) and with a depth as that of Eq. (6) gives a dispersive real potential of the form

$$
\Delta V(r, E)=\Delta V(r) \Delta V(E),
$$

being

$$
\Delta V(r)+\left\{1+\exp \left[\left(r-R_{i}\right) / a_{i}\right]\right\}^{-1}
$$

and

$$
\begin{aligned}
\Delta V(E)=\frac{1}{\pi}[ & \left(a_{1}+b_{1} E\right) \ln \frac{\left|E_{c}-E\right|}{\left|E_{i}-E\right|} \\
& \left.+\left(a_{2}+b_{2} E\right) \ln \frac{\left|E_{f}-E\right|}{\left|E_{c}-E\right|}\right],
\end{aligned}
$$

it being well understood that the geometrical parameters $r_{i}$ and $a_{i}$ are energy independent. The constants $a_{1}, b_{1}$, $a_{2}, b_{2}, E_{i}, E_{c}$, and $E_{f}$ are determined from lineal least square fits of the empirical depths for each system. Figure 2 shows these fits for the elastic scattering of ${ }^{16} \mathrm{O}$ by

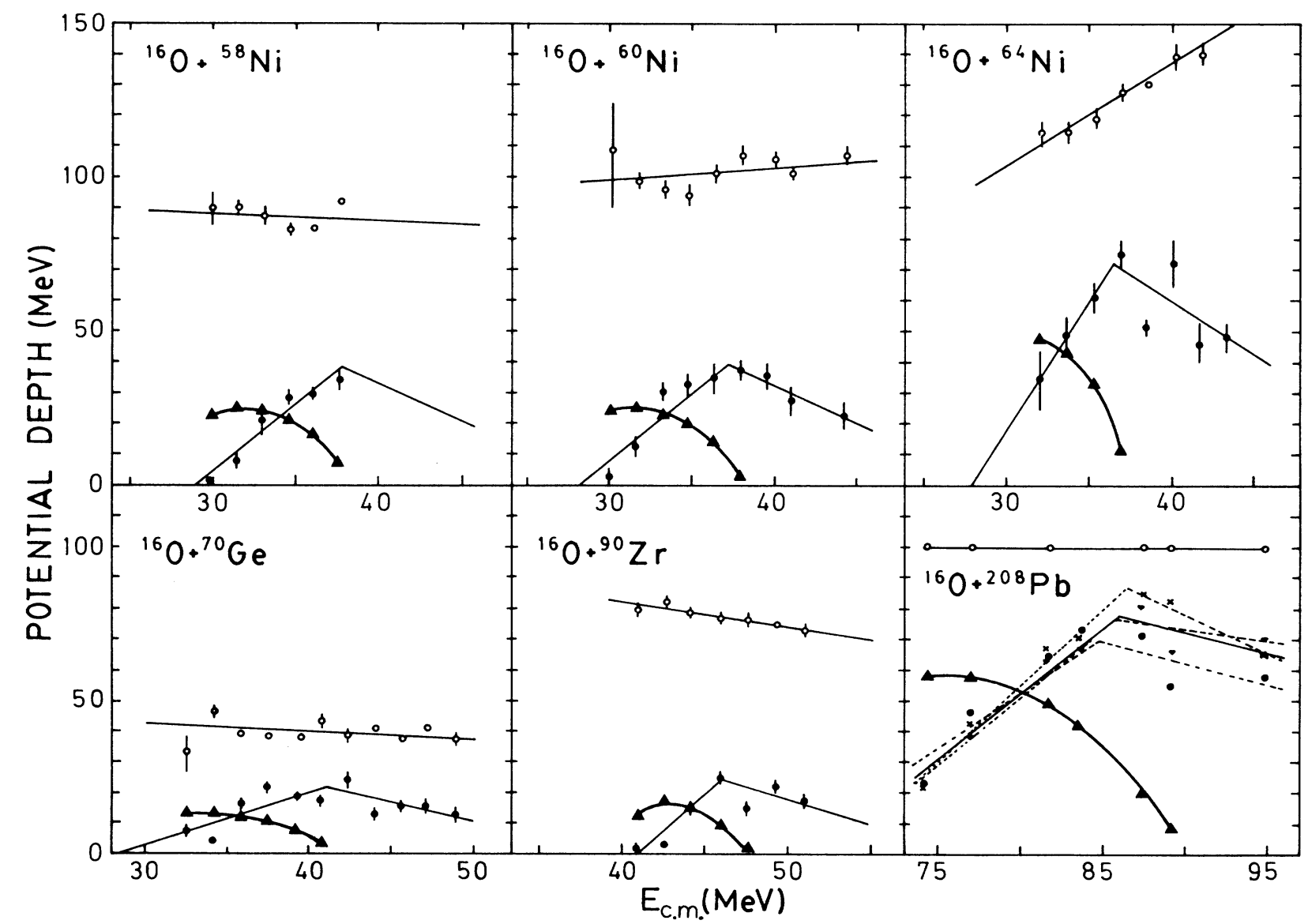

FIG. 2. The values of real (open circles), imaginary (full circles and other symbols in the last case), and dispersive (triangles) potential well depths, as functions of center of mass energy for the elastic scattering of ${ }^{16} \mathrm{O}$ from ${ }^{58,60,64} \mathrm{Ni}(\operatorname{Ref} .4),{ }^{70} \mathrm{Ge},{ }^{90} \mathrm{Zr}(\operatorname{Ref} .5)$, and ${ }^{208} \mathrm{~Pb}$ (Ref. 6). The straight line fit to the real and imaginary depths is shown. The full heavy line joins the dispersive depths. 
different target nuclei. The data are taken from Ref. 4 $\left({ }^{16} \mathrm{O}+{ }^{58,60,64} \mathrm{Ni}\right)$, Ref. $5\left({ }^{16} \mathrm{O}+{ }^{70} \mathrm{Ge},{ }^{90} \mathrm{Zr}\right)$, and Ref. 6 $\left({ }^{16} \mathrm{O}+{ }^{208} \mathrm{~Pb}\right)$. The error bars are a measure of the uncertainties associated with the ambiguity of the optical model parameters. $^{4,5}$ The reported points in Fig. 2 are those around and above the Coulomb barrier.

In the case of ${ }^{16} \mathrm{O}+{ }^{58} \mathrm{Ni}$ the same values of $a_{2}$ and $b_{2}$ as for ${ }^{16} \mathrm{O}+{ }^{60} \mathrm{Ni}$ are adopted since there are no empirical depths at the higher energies and not much difference is expected in principle between these two systems. The geometry parameters for the first five systems reported in Fig. 2 were the same for the real and the imaginary parts and energy independent., ${ }^{4,5}$ These are $r=1.22 \mathrm{fm}$ and $a=0.50 \mathrm{fm}$ for ${ }^{16} \mathrm{O}+{ }^{58,60,64} \mathrm{Ni}, r=1.25 \mathrm{fm}$ and $a=6.0$ fm for ${ }^{16} \mathrm{O}+{ }^{70} \mathrm{Ge}$, and $r=1.25 \mathrm{fm}$ and $a=0.50 \mathrm{fm}$ for ${ }^{16} \mathrm{O}+{ }^{90} \mathrm{Zr}$. For the case of ${ }^{16} \mathrm{O}+{ }^{208} \mathrm{~Pb}$ Videbaek et al. ${ }^{6}$ offer three different sets of Woods-Saxon parameters with the same geometry for the real and imaginary potentials and with a common real depth of $100 \mathrm{MeV}$. Although in this case the radius parameter of each set varies slightly with energy, it was not considered in the integration of the dispersion relation. For the imaginary depths an average value for the lineal fits of the three sets has been taken, $a_{1}=-294.7 \mathrm{MeV}, b_{1}=4.33, a_{2}=195.7 \mathrm{MeV}$, and $b_{2}=-1.37$ that give the following values of the energies: $E_{i}=68 \mathrm{MeV}, E_{f}=143 \mathrm{MeV}$, and $E_{c}=86 \mathrm{MeV}$. Once the constants of expression (6) are determined from the lineal fits, the dispersive potential depths are calculated with Eq. (9). The result is displayed in Fig. 2 by the heavy line joining the triangular points. As can be seen, in every case the part of the empirical real depth attributed to the dispersive contribution is very important, especially when the energy approaches the Coulomb barrier. Only a few $\mathrm{MeV}$ above the barrier the importance of this term decreases rapidly, and for a certain energy the dispersive potential changes its sign. Since, in general, the increasing part of the depths (in absolute value) with the energy is better fitted by a straight line than the decreasing one, expression (9) has been calculated for different values of the constants $a_{2}, b_{2}$, and $E_{f}$. The results do not change significantly, as can be expected from the results of Mahaux, Ngô, and Satchler. ${ }^{16}$

In order to see if the addition of the dispersive potential to the folding real potential can avoid the renormalization of this one, the system ${ }^{16} \mathrm{O}+{ }^{208} \mathrm{~Pb}$ was chosen. Figure 3 shows the empirical Woods-Saxon potentials of Videbaek et al. $^{6}$ for two energies, $E=74.3$ and $83.6 \mathrm{MeV}$ (80 and $90 \mathrm{MeV}$ laboratory), at the strong absorption radius. This is defined here as the point where potentials with different diffusivities $(0.45,0.50$, and $0.55 \mathrm{fm})$ cross each other. The long dashed line represents the double folding potential in that region calculated with the method of Ref. 3 . The short dashed line is the dispersive potential (7) constructed with a geometrical part (8) corresponding to the parameter set of diffusivity $0.50 \mathrm{fm}$ of Videbaek et al. ${ }^{6}$ and a depth calculated with expression (9). Finally, the

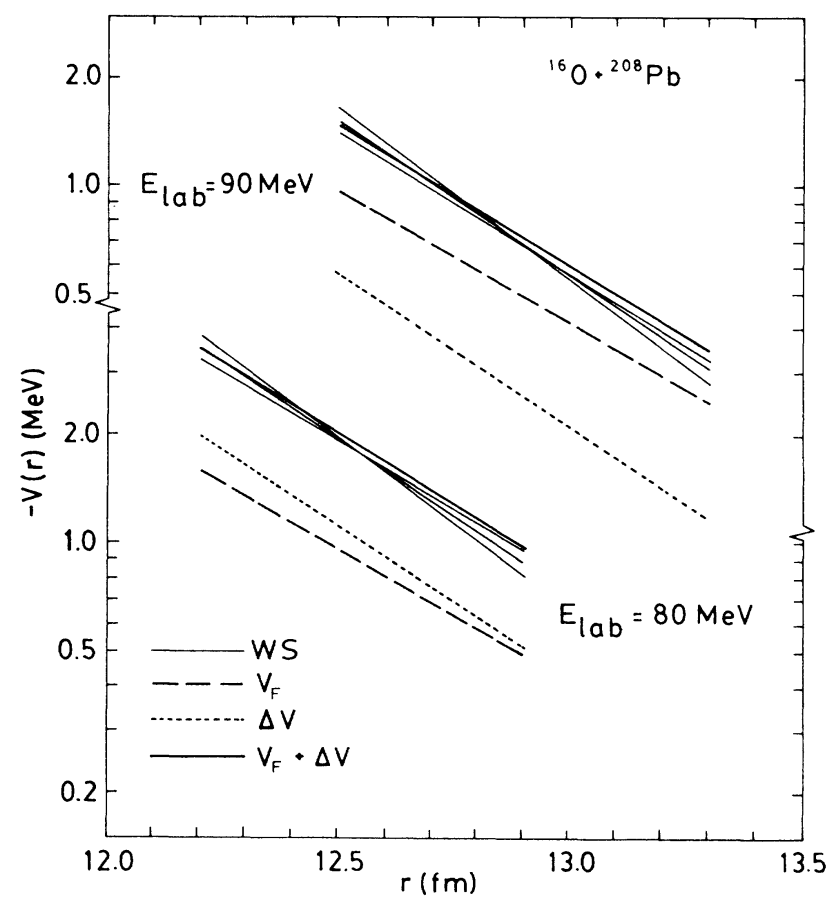

FIG. 3. The real nuclear potentials as a function of the radius in the tail region for the elastic scattering of ${ }^{16} \mathrm{O}$ by ${ }^{208} \mathrm{~Pb}$ at 80 and $90 \mathrm{MeV}$ of laboratory energy. The three lines crossing each other correspond to best fit Woods-Saxon potentials with diffusivities $0.45,0.50$, and $0.55 \mathrm{fm}$ as taken from Videbaek et al. (Ref. 6). The long dashed line is the double folding potential (Ref. 3). The short dashed line is the dispersive contribution to the real potential (see the text) and the full heavy line is the sum of the last two potentials.

heavy continuous line is the sum of these two potentials. As it can be seen, the agreement with the empirical potentials is remarkable for the rough approximations that have been made.

In conclusion, the causality principle that induces a dispersion relation connecting the imaginary part of the optical potential with the real one can explain the anomalies presented by the microscopically calculated real potential between ${ }^{16} \mathrm{O}$ and several target nuclei. In this light, the reanalysis of early obtained empirical potentials shows the part of the real potential that can be attributed to couplings of the elastic with nonelastic channels at energies close to the Coulomb barrier. These couplings can be included in a global way in the dispersion relation.

A careful reading of the manuscript by G. M. Madurga is acknowledged. This work was partially supported by the Spanish Comisión Asesora de Investigación Cientifica y Técnica under Contract No. 2868-83.
${ }^{1}$ M. Lozano and G. Madurga, Nucl. Phys. A334, 349 (1980).

${ }^{2}$ G. R. Satchler and W. G. Love, Phys. Rep. 55, 183 (1979).

${ }^{3}$ F. J. Viñas, M. Lozano, and G. Madurga, Phys. Rev. C 23, 780 (1981).
${ }^{4}$ L. West, Jr., K. W. Kemper, and N. R. Fletcher, Phys. Rev. C 11, 859 (1975).

${ }^{5}$ A. W. Obst, D. L. McShan, and R. H. Davis, Phys. Rev. C 6, 1814 (1972). 
${ }^{6}$ F. Videbaek, R. B. Goldstein, L. Grodzins, S. G. Steadman, T. A. Belote, and J. D. Garret, Phys. Rev. C 15, 954 (1977).

${ }^{7}$ M. V. Andrés, J. M. Quesada, M. Lozano, and G. Madurga, Nucl. Phys. A443, 380 (1985).

8J. Gomez-Camacho, M. Lozano, and M. A. Nagarajan, Nucl. Phys. A440, 543 (1985).

9 J. S. Lilley, B. R. Fulton, M. A. Nagarajan, I. S. Thompson, and D. W. Banes, Phys. Lett. 151B, 181 (1985).

${ }^{10}$ A. Baeza, B. Bilwes, R. Bilwes, J. Diaz, and J. Ferrero, Nucl. Phys. A419, 412 (1984).
${ }^{11}$ I. J. Thompson, M. A. Nagarajan, J. S. Lilley, and B. R. Fulton, Phys. Lett. 157B, 250 (1985).

${ }^{12}$ S. C. Pieper, M. J. Rhoades-Brown, and S. Landowne (unpublished).

${ }^{13}$ A. A. Ioannides and R. S. Mckintosh (unpublished).

${ }^{14}$ M. A. Nagarajan, C. Mahaux, and G. R. Satchler, Phys. Rev. Lett. 54, 1136 (1985).

${ }^{15}$ C. Mahaux and R. Sartor, Nucl. Phys. A460, 466 (1986).

${ }^{16}$ C. Mahaux, H. Ngô, and G. R. Satchler, Nucl. Phys. A449, 354 (1986). 